\title{
Systemvorteile faserverstärkter Thermoplast-Teile
}

Nach gerade einmal zweijähriger Vorbereitungszeit ging mit der ITHEC (International Conference and Exhibition on Thermoplastic Composites) in Bremen Ende Oktober ein neuer Kongress mit dem Themenschwerpunkt faserverstärkter Verbundwerkstoffe an den Start. Ziel der Veranstaltung, die von der Messe Bremen in Kooperation mit dem Faserinstitut Bremen (FIBRE) organisiert wurde und die künftig alle zwei Jahre stattfinden soll, ist es, Anwenderindustrien die Systemvorteile faserverstärkter Thermoplast-Teile näher zu bringen.

Für den Initiator der Veranstaltung, Professor Axel S. Hermann, Institutsleiter des Faserinstituts, Vorstandsvorsitzender des CFK-Valley Stade und zugleich Geschäftsführer der Airbus Tochter Composite Technology Center (CTC), ist es „eigentlich ein Wunder, dass es ein solches Spezialforum bisher weltweit nicht gab". Hermann sieht Faserverbundwerkstoffe auf Basis einer Thermoplast-Matrix als Schlüssel, Gewichtseinsparungen etwa im Bereich der Automobilindustrie wirtschaftlich und in großen Stückzahlen zu realisieren. Die meisten Produktentwickler und Fertigungstechniker dächten indes in erster Linie an faserverstärkte Kunststoffe mit duroplastischer Matrix, wenn sie den Begriff CFK lesen, stellte Hermann fest. Gegenüber CFK mit duroplastischer Matrix haben Thermoplaste jedoch gravierende
Vorteile, wie in 26 Vorträgen und der begleitenden Ausstellung mit 25 Ausstellern unterstrichen wurde. Dazu gehören in erster Linie die kürzeren Zykluszeiten, mit denen sich Bauteile im Minutentakt produzieren lassen. Während die duroplastischen Matrixsysteme auf Harzen basieren, die zwischen 6 min und mehreren Stunden aushärten müssen, könnten faserverstärkte thermoplastische Bauteile im Minutentakt produziert werden. Ein weiterer Vorteil sei die größere Prozessvielfalt. Eine Kombination aus Spritzgießen und Thermoformen, etwa das Hinterspritzen von Organoblechen, ermögliche komplexe Geometrien.

Das FIBRE stellte Prozessentwicklungen vor, die zu belastungsgerechten verschnittarmen Preforms gehen. Durch den Einsatz von trockenen, nicht vorkonsolidierten thermoplastisch gestickten Hybridpreforms könne im Vergleich zu vorkonsolidierten Organoblechen eine Kostenreduktion auf Material- und Prozessseite erreicht werden, hieß es. Schließlich sprächen die bessere Recycelbarkeit, eine einfache Lagerung sowie attraktive Verbindungstechniken für Thermoplaststrukturen. Ließen sich Thermoplaste grundsätzlich bearbeiten wie Bleche, so seien es gerade diese neuen Verbindungsmöglichkeiten, mit denen sich erhebliche Kostenvorteile erzielen ließen.

Wenngleich sich die ITHEC schwerpunkt- mäßig mit kohlenfaserverstärkten Strukturen mit thermoplastischer Matrix beschäftigte, wurde auch die Suche nach Alternativen zu den vergleichsweise teuren Carbonfasern thematisiert. Zwar seien Carbonfasern für hoch belastete und hochfeste Strukturen nach wie vor erste Wahl, betonte Professor Hermann. Wer jedoch in den Markt höre, wisse auch, dass die Anwendermärkte bei den verwendeten Fasern einen Kilopreis von sechs bis sieben Euro statt der bislang üblichen 15 bis 20 Euro wünschten und es zudem eine deutliche Forderung nach hochgradig recycelbaren Faser-Matrix-Kombinationen gebe. Hermann sieht eine Chance für Lösungen, die unabhängig vom Vorprodukt Erdöl machten, etwa Fasern, die auf Lignin basieren, also im weitesten Sinne auf Holz oder Zellulosefasern. Dies sei noch Zunftsmusik, so der Experte, doch das Denken in Kunststoff erfordere neue Facetten.

Die ITHEC-Premiere verfolgten knapp 280 Teilnehmer, die zu $80 \%$ aus der Industrie kamen. Damit war ein wichtiges Ziel der Veranstalter erreicht, die eben keine neue Wissenschaftsplattform schaffen, sondern eine Verbesserung der Prozesskette erreichen wollen. „Wir brauchen die Nachfrage der OEM, um die thermoplastische Produktion voranzutreiben und den Transfer in den Leichtbau zu fördern", so Faserinstituts-Chef Hermann.

$\mathrm{cg}$

TENCATE UND BASF

\section{Strategische Allianz zu thermoplastischen Verbundwerkstoffen}

Tencate Advanced Composites (NL) und die BASF wollen gemeinsam thermoplastische Verbundwerkstoffe für die automobile Großserienproduktion entwickeln, herstellen und vermarkten. Zu diesem Zweck sind beide Unternehmen eine strategische Allianz eingegangen. Ziel der Partnerschaft ist, den Fahrzeugherstellern maßgeschneiderte Lösungen aus Hochleistungsfaserver- bundstrukturen zur Verfügung zu stellen, die eine weitere Reduzierung des Fahrzeuggewichts möglich machen.

Die BASF bringt ihr Know-how in der Herstellung und Veredelung (Formulierung) von thermoplastischen Kunststoffen ein und entwickelt spezielle Varianten ihres Ultramid- (PA), Ultradur- (PBT) und Ultrason- (PESU) Sortiments, heißt es in einer
Mitteilung beider Unternehmen. Tencate trage mit seiner Expertise in der Herstellung der Faserverbundwerkstoffe zur Allianz bei. Vereint wollen sich sich beide Firmen den auf diesen Kunststoffspezialitäten basierenden Faserverbundwerkstoffen und Halbzeugen für den Automobilbau (UD-Tapes, Prepregs und Laminate) widmen. 